\title{
Spectrum Efficiency Improvement Based on the Cognitive Radio Management
}

\author{
Jamal Raiyn \\ QRC-Qasemi Research Center, Alqasemi College, Baka Alqarbiah, Israel \\ Email: rayan@qsm.ac.il \\ Received November 3, 2009; revised December 5, 2009; accepted January 19, 2010
}

\begin{abstract}
Interference and delay are considered as the major reasons limiting the capacity and increasing the new call blocking probability in cellular system. In this paper we introduce a novel strategy based on cognitive radio. Cognitive radio is defined as a radio or system that senses its environment and can dynamically and autonomously change its transmitter parameters based on interaction with the environment in which it operates, such as maximize throughput and reduce interference. The goal of the use of cognitive radio is to improve the spectrum efficiency in cellular system. Spectrum management based on radio cognitive plays thereby an important role to increase the capacity of the radio systems and spectrum utilization, especially in the context of open spectrum.
\end{abstract}

Keywords: Cognitive Radio, Handover, Social Agent

\section{Introduction}

In this paper we introduce cognitive radio approach that is expected to perform more significant role in the view of efficient utilization of the spectrum resources in the future wireless communication networks. The spectrum utilization efficiency is defined as the ratio of information transferred to the amount of spectrum utilization. Our approach is reactive approach, in that it enables, via negotiation, learning, reasoning, prediction, active sense, identification, changes in the base station's parameter to meet the new services requirements in modern wireless networks and future challenges in cellular systems. A major challenge with cognitive radio approach is to be done in near real-time and to keep up with an ever changing RF environment without overly computationally complex. Various resource allocation strategies are proposed to optimize the resource allocation in cellular system by reducing the call blocking probability $[2,3$, $5,6,8]$ in cellular systems. The call blocking probability is often measured in terms of two blocking probabilities, the arriving call blocking probability, and the handover blocking probability. Analyses and studies in [9-11] show that the call blocking probability in handover is caused by two main parameters, interference and delay. Interference leads to missed and blocked calls due to errors in the digital signaling. Between transmitter (Base Station, BS) and receiver (Mobile Station, MS), the channel is modeled by several key parameters. These pa- rameters vary significantly with the environment (urban, rural, mountains). There are different type of interference that when not minimized, decreases the ratio of carrier to interference power at the periphery of the cells, causing diminished system capacity, more handover $[1,4]$, and more dropped calls. To reduce the handover blocking probability in cellular systems has been proposed various schemes as, prioritized handover schemes and handover with queueing [4]. In some application fields like realtime communication and industrial automation is needed to ensure a seamless and lossless handoff. Which means the handover latency should be zero. For efficient handover management, Handover is a basic mobile network capability for dynamic support of terminal migration. Handover Management is the process of initiating and ensuring a seamless and lossless handover of a mobile terminal from the region covered by one base station to another base station. In this paper we consider the handover call blocking probability in cellular systems.

The paper is organized as follows: Section 2 introduces the system model and the social agent strategies. Section 3 discuss and analyze the handover call blocking probability based on simulation results and Section 4 concludes the paper.

\section{Problem Description}

Over the last two decades, the demand for mobile host 
and multimedia services increased rapidly. One of the biggest challenges in supporting multimedia applications in cellular systems is to fulfill the mobile user demand and satisfy his preferences under the constraint of the limited radio bandwidth, and to utilize the limited spectrum availability to meet the increasing demand for mobile service. Some of the most often used methods to increase the spectral efficiency are resource allocation schemes. Various channel allocation schemes have been introduced to provide Quality of Service (QoS) [14,15, 16] and efficient channel utilization in cellular networks. There are many parameters to measure the QoS of a network. These include throughput, latency, service availability etc. The blocking probability is one of the most important QoS parameters. Since users are mobile, the QoS of wireless networks are often measured in terms of two probabilities: the new arriving call blocking probability and the handoff blocking probability. Hence, this paper deals with the main issue: How to allocate resources (e.g. frequency channels) to radio ports of a wireless system (e.g. cells in a cellular mobile network) that can improve the traffic performance of network (e.g. lower blocking probability in voice networks, lower latency in data networks etc.)?

In the modern data communication systems, we consider the power transmission as the resources. An increase of transmission power enables the increase of data rate and subsequently shorter transmission time. On the other hand, an increased transmission power causes more interference in neighboring cells. One of the physical measures of RF channel quality is the carrier-to-interference or $\mathrm{CI} R$. This ratio is logarithmically proportional to the signal quality enjoyed by the receiver of the signal. The larger the $C / I$ ratio, the better the channel quality is. If the measured $C / I$ falls below a certain level, $C I R_{\min }$, which depends on system type and operator requirements, the mobile should be in the coverage region of another cell and a call handoff should be performed. The interior of the cell should provide $C / I$ ratio which exceed this level, unless the mobile is located in an RF coverage "hole." In general, for a feasible solution for the resource managements in the cellular systems must be satisfied four requirements as follows:

- Coverage requirement: each of the MS locations must be able to connect at least to one base station and the received signal strength must exceed a given level $P_{\min }$.

- Transmitter power requirements: the transmitted power of the mobile stations in the system must not exceed a maximum level $P_{\max }$.

- Downlink SIR requirement: each downlink SIR must exceed a threshold of SIR ${ }^{\text {th }}$.

- Uplink SIR's requirement: each uplink SIR must exceed a threshold of SIR ${ }^{\text {th }}$.

In this case, we consider the problem of minimizing total transmitter power. To minimize the total transmit power, subject to the constraint that each transmitter/ receiver attain a maximum allowed outage probability i.e., a minimum allowed quality of service and subject to limits on the individual transmitter power, we formulate the problem as follows:

$$
\text { minimize } P_{1}+\ldots+P_{n}
$$

Subject to $P_{i}^{\min } \leq P_{i} \leq P_{i}^{\max }, i=1, \ldots, \mathrm{n}$

Here, $P_{i}^{\min }$ and $P_{i}^{\max }$ are the minimum and maximum transmitter power for the transmitter $i$. To handle the resource allocation problem in cellular systems we introduce the cognitive radio scheme. For the channel allocation problem, the main goal is to bring more distribution of control. In cognitive radio scheme, each cell (base station) is managed by an agent. The resource in the system will be distributed and allocated through interaction between agents and limited to the domain of the agents. In other words, the channel allocation problem can be restricted to one domain, so that the agents can be placed in the base stations.

\section{Power Control Based on CR}

Each cell $i$ can acquire amount $i$ while the level in the cell is denoted by $h_{i}$ (the demand of channel in each cell depends on the arrival call in the cell). We assume, that the mass flow of resource in the cell $i$ is proportional to the value position $u_{i}$ (call arrival rate). And we consider that the resources in the system are limited. The vector of the required set points of the levels, (to meet the call arrival rate), are given by $h_{s}$. We further assume that the equilibrium point $\left(u_{0}, h_{s}\right)$ is known (channel allocation). The control task is the regulation of disturbances (plus the interference) of the resources in the cells. Each cell is considered as a single subsystem and associated with an agent. The input variable of each agent $i$ is the deviation $\Delta h_{i}(k)$ of the resource amount.

$$
\Delta h_{i}(k)=h_{i}-h_{s}
$$

The output variable is the value position

$$
u_{i}(k)=\Delta u_{i}(k)+u_{0}
$$

which is the sum of the deviation $\Delta u_{i}(k)$ and the equilibrium position $u_{0}$. Since the equilibrium position is known, the agent only has to compute the output variable $\Delta u_{i}(k)$. Since the amount of the resources that flows into the cell is limited, the absolute Value $\left|\Delta u_{i}(k)\right|$ is considered as the resource variables. Now each agent can either play the role of the consumer or the producer. A positive deviation $\Delta h_{i}(k)$ means that the cell "needs" some more resource to reach the required level (i.e. to allocate all calls).

$$
\Delta h_{i}(k)=h_{i}-h_{s}>0 . \quad \text { i.e., } \quad h_{\mathrm{i}}>h_{\mathrm{s}}
$$


Thus, the related agent can be declared as a consumer, which has to acquire a certain amount of the resource. This demand of consumer $i$ at instance $k$ is denoted by $r_{D i}(k)$. The goal of the consumer is to maximize his utility, (i.e. by reducing the call blocking probability). $J_{C i}(k)$ with

$$
\frac{J_{C i}(k)}{r_{D i}(k)}=\Delta h_{i}(k)-r_{D i}(k) * I(k)
$$

The relative utility is a composition of two terms. The first term takes into account that the utility of the cell from getting some resource increases with increasing deviation $\Delta h_{i}(k)$. The second term denotes the consumer's expenditure (allocation) with respect to his interference $I(k)$. Its utility decreases with increasing expenditures. The minimization of the call blocking probability $J_{C i}(k)$ leads to the consumer's demand:

$$
r_{D i}(k)=\Delta h_{i}(k) * \frac{1}{I(k)}
$$

The demand is a function of the interference. An increasing of the interference leads to a decreasing demand. In the case of a negative deviation $\Delta h_{i}(k)$, the cell contains too much free resources. Hence, the related agent is considered as a producer which wants to gift this resource to the consumers with the goal to maximize system capacity/efficiency and its profit $J_{p i}(k)$, denoted by

$$
\frac{J_{p i}(k)}{r_{S i}(k)}=\left|\Delta h_{i}(k)\right| * I(k)-r_{S i}(k)
$$

The relative profit contain two terms; The first term takes into account that the utility of the producer increases with his income and with increasing deviation $\left|\Delta h_{i}(k)\right|$. The second term is production costs that are assumed to be proportional to the supply. The maximization of $J_{p i}(k)$ leads to the supply of producer agent $i$ :

$$
r_{S i}(k)=\left|\Delta h_{i}(k)\right| * I(k)
$$

One problem occurs: If all agents are producers or all agents are consumers. However, there is always nominal channels/resources in the cells (nominal channel can be used only from own cell). Therefore, obviously have a permanent producer, which is independent of the amount of resource (swapping method). Assuming $n$ coupled cells, the actual number of producers at the instance $k$ is $m$ while the actual number of consumers is $q$ with $n=m$ $+q$. Each consumer agents computes his demand while each producer agents computes his supply. All functions are transmitted to an auctioneer agent using the communication network (the auctioneer could be the distributor agent, $(D)$. The task of the auctioneer is the computation of the equilibrium using the constraint that the sum of all demands has to equal the sum of all supplies:

$$
r_{D p}(k)+\sum_{i=1}^{q} r_{D i}(k)=r_{S p}(k)+\sum_{j=1}^{m} r_{S i}(k)
$$

Hence the output variables $\Delta u_{i}(k)$ are

$$
\begin{aligned}
& \Delta u_{i}(k)=\Delta h_{i}(k) * \frac{1}{I(k)}, i \text { consumer. } \\
& \Delta u_{i}(k)=\Delta h_{i}(k) * I(k), i \text { producer. }
\end{aligned}
$$

\section{- Power control}

The power control can raise the network capacity. Some power control algorithms $[17,18,19,20]$ based on the idea of balancing the SIR of all radio links have been introduced, but the final SIR achieved by those algorithms may be unsatisfactory for some of the links. Some calls must be dropped in order to keep the SIR of other calls higher than the predefined threshold value. Obviously, the efficiency of radio resource management is dependent on the channel assignment and the power control. The combination of DCA and power control to obtain some substantial capacity gains has been reported in [22], however, because no channel pre-selection is done before the channel probing procedure, inadvertent dropping of calls caused by originating calls can occur so often that all unsuccessful (blocked or dropped) calls are unintentionally dropped calls and not blocked calls. In addition, an exhaustive search and too frequent intra-cell handoff access will decrease the system capacity and make the algorithms difficult to implement in real networks. Here, a cognitive radio algorithm with power control is proposed. The power that is transmitted both from the mobile equipment and from the base station has a far-reaching effect on efficient usage of the spectrum. Power control is an essential feature in mobile networks, in both uplink and downlink. When a mobile transmits high power, there is enough margin in the critical uplink direction. But it can cause interference to other subscriber connections. The power of the signal transmitted by the base station antenna should be kept to a level above the required threshold without causing interference to the mobiles. Mobile stations thus have a feature such that their power of transmission can be controlled. This feature is generally controlled by the BSS. This control is based on an algorithm that computes the power received by the base station and, based on its assessment, it increases or decreases the power transmitted by the mobile station. The signal power at the ith receiver is given by $G_{i i} F_{i i} P_{k}$ [21], and the total interference power is given by $\sum_{k \neq i} G_{i k} F_{i k} P_{k}$

The SIR of the $i$ th receiver (or transmitter) is given by

$$
S I R_{i}=\frac{G_{i i} F_{i i} P_{k}}{\sum_{k \neq i} G_{i k} F_{i k} P_{k}}
$$




$$
\operatorname{SIR}_{i}=\frac{G_{i i} F_{i i} P_{k}}{I_{i}}
$$

We assume that the QoS requested is provided when the SIR exceeds a given threshold $S I R^{\text {th }}$. The outage probability of the $i$ th receiver/transmitter pair is given by

$$
\begin{aligned}
O_{i} & =\operatorname{Pr} o b\left(S I R_{i}\right) \leq S I R^{t h} \\
& =\operatorname{Pr} o b\left(G_{i i} F_{i i} P_{i} \leq S I R^{t h} \sum_{k \neq i} G_{i k} F_{i k} P_{k}\right)
\end{aligned}
$$

The outage probability $O_{i}$ can be interpreted as the fraction of time the $i$ th transmitter/receiver pair experiences an outage due to fading. Note that the in our expression for $O_{i}$, we take into account statistical variation of both received signal power and received interference power. We now consider the market method to regulate the SIR. By ignoring all statistical variation of both signal and noise power, the signal power at the $i$ th receiver is then $G_{i i} P_{i}$ and the interference power at the receiver is given by $\sum_{k \neq i} G_{i k} P_{k}$. Then the SIR at the $i$ th receiver is given as

$$
\operatorname{SIR}_{i}^{m}=\frac{G_{i i} P_{i}}{\sum_{k \neq i} G_{i k} P_{k}}
$$

We interpret $\operatorname{SIR}_{i}^{m}$ as follows: this is what the signal-to-interference of the ith transmitter/receiver pair would be, if the fading state of the system where $F_{l}=\ldots$ $=F_{n}=1$. We also define

$$
S I R^{m}=\min _{i} S I R_{i}^{m}=\min _{i} \frac{G_{i i} P_{i}}{\sum_{k \neq i} G_{i k} P_{k}}
$$

which is the minimum SIR of the system over all transmitter/receiver pairs. Like the outage probability $O$, the $S I R^{m}$ gives a figure of merit for the system and power allocation. We define the market regulation/control method of the system and power allocation as the ratio of the market control SIR to the signal-to-interference reception threshold

$$
M C A=\frac{S I R^{m}}{S I R^{t h}}=\min _{i} \frac{G_{i i} P_{i}}{S I R^{t h} \sum_{k \neq i} G_{i k} P_{k}}
$$

There is a relation between MCA and $O$ : when MCA is large (which means that the SIR, ignoring statistical variation, is well above the minimum required for reception), we should have small $O$. Let

$$
\delta_{i}=\frac{G_{i k}}{I_{i}}
$$

denote the channel variation. $\delta_{i}$ will be estimated and predicted in the proposed power control scheme.
$I_{i}=\sum G_{i k} F_{i k}$ presents the received interference. $G_{i k}$ is the link gain from mobile station $k$ to base station $i$. Suppose, that the highest transmitted power allowed is $P^{\max }$ and that the lowest transmitted power allowed is $P^{\min }$. The social agent that SNR oriented uses the following technique to regulate the SNR between the cells. The proposed technique operates in the following way:

- For any cell, two tiers of cells are considered as interfering cells. The channel state information (allocating or releasing) of each cell is locally exchanged to its interfering cells. Every cell maintains a list of the cost for all channels. The cost function is used to decide the cost of a channel. The cost of a channel in a cell is updated (increased or decreased) in real time if a co-channel call is accepted or terminated (dropping and departure) in one of the cell's interfering cells.

- When a call arrives in a cell, the free channel with highest priority (lowest cost) is chosen for call setup and the call power probing process is activated. The procedures of the power probing for a new (or handoff) call are:

1) Assigning the minimum transmitted power Pmin to the new call $p$.

2) Measuring the SIR value $\gamma_{p}$ of the call.

3) If $\gamma_{p}<\gamma$, adjusting the power of the call and going back to step 2 ; if $\gamma_{p} \geq \gamma$, and $P^{\min } \leq P_{p}(k) \leq P^{\max }$, this call is admitted into service with this power and the call power probing process is ended.

4) If a power cannot be found in the range of $\left[P^{\min }\right.$, $\left.P^{\max }\right]$ with which the SIR value $\gamma_{p} \geq \gamma$, or the probing iteration number is larger than a pre-assigned value, the probing is moved to the next highest priority channel. Actually, an exhaustive searching is not allowed in a system. Hence, we prescribe that if four channels have been evaluated, but the SIR requirement is still not satisfied, the call is blocked.

5) If a call is in service, the power control algorithm is used to maintain its quality. Each base station monitors its own served calls at some time interval. We assume that all base stations are synchronized (actually the algorithm works asynchronously either). When the SIR of a call falls below the target value, the power control procedure is requested. However, if the maximum transmitted power is requested or the number of iterations of power level adjustment is larger than the allowed value, but the SIR is still below a specified value (e.g., the call dropping threshold value), the handoff procedure is requested. The "call set-up" procedure will begin to search for a channel for handoff. If a channel is found, the call is moved to this channel. Otherwise, the call is dropped.

\section{System Model}

A cellular network consists of an array of cells. We par- 
tition the cellular network to clusters. For each cell we set a social agent, as depicted in Figure 1. By using radio cognitive approach we aim to achieve an optimal network capacity, minimizing interference to other signals and to reduce messages complexity and channel acquisition delay that are considered the main reasons to block the new calls. A radio cognitive approach may be able to sense the current spectral environment, and have information of past transmitted and received packets along with the power, bandwidth, and modulation. By considering all this, it makes better decisions about how to best optimize for some overall goal. Under heavy traffic load, and if a vacant channel is not found, the social agent then tries to obtain an exclusive channel by optimization of channel distribution based on iterative swapping scheme [23]. In which the social agent changes the channel distribution anew. We partition the set of channels into active and passive channels. The active channels are defined as the channels, which can be used by own cell. Furthermore the active channels can be simultaneously used in different cells without any interference if they are a minimum reuse distance $\left(D_{\min }\right)$ apart from each other [23]. The passive channels are defined as the channels, which can be used by neighbor cells. Furthermore, the free channel will be expressed as 1 and the busy channel will be expressed as 0 . The set of channels are classified and assigned uniform to cells in real-time.

\subsection{Social Agent Negotiation Strategy}

Compared to the traditional negotiation strategy that offers high messages complexity, we are interested in ap-

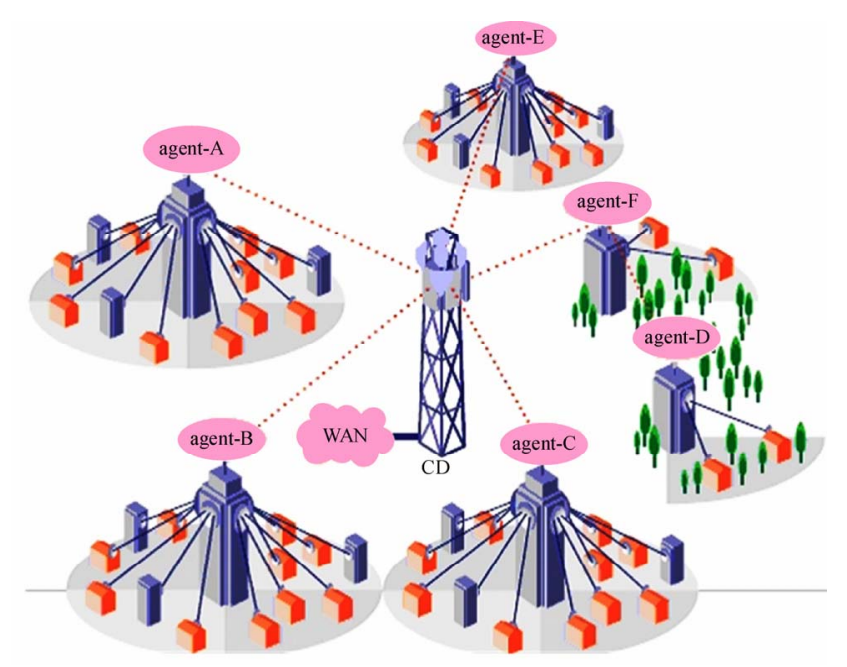

Figure 1. System model. plications where negotiation between social agents serves to solve the resource allocation problem in cellular systems. Furthermore we anticipate concern the feasibility of reaching an allocation of resources that is optimal from a social point of view. Social agents often need to interact in order to improve their performance. One type of interaction that is gaining an increasing interest is dynamic negotiation. The goal of negotiation is the maximization of the utility of a future decision. In distributed dynamic environment, each cell has an objective that specifies its intention to acquire a free channel for call establishment. That objective should be achieved in a certain amount of time, specified by a deadline. Negotiation stops when this deadline is reached.

\subsection{Social Agent Decision Strategy}

In this phase the agent deals with handover request as illustrates in Figure 2. The social agent's decision about the handover process is focused on quality of service requirements (e.g. signal power, signal-to-noise ratio and delay). The signal-to-noise ratio (SNR or S/R) defined as the ratio of a signal power to the noise power corrupting the signal. The social agent estimates the SNR and then determines to carry out the handover or not. Furthermore the agent collects information about the adjacent cells. Based on of the collected information from the adjacent cells, the agent determines the next handover cell. The handover based on SNR can be divided into two main categories:

1) The first scenario is based on received SNR from the base station only. This method decides handoff when the SNR from current station is smaller than another station. This kind of method is simple but will take place repeated or unnecessary handoff.

2) The second scenario is based on relative SNR with threshold. In this approach, handoff is initialed when the average SNR falls below a certain threshold value. This method can avoid unnecessary handoff when the current station signal is still satisfactory.

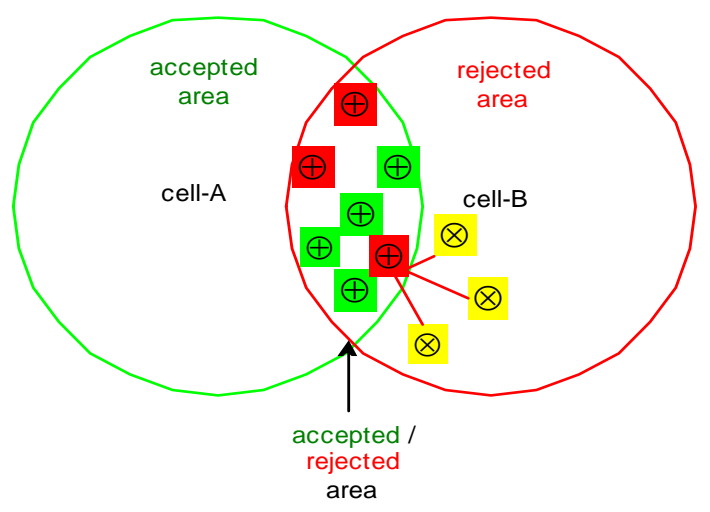

Figure 2. Blocking probability area. 


\subsection{Social Agent Reasoning Strategy}

The radio approach draws its decision based on employing a cost function [24]. The resource agent allocates to a request, the channel of which the cost function is minimal. The cost function basically computes the interference level. The cost functions can be collectively expressed in a general expression:

$$
J_{k}=\sum_{i \in I_{c}}\left(C_{k i} q_{k i}\right)
$$

where $J_{k}$ is the channel interference cost unit for the $k$-th channel, $I_{c}$ denotes the set of co-channel interference cells related to cell $C . C_{k i}$ denotes the binary status of $I_{c}$ which signifies that

$$
C_{k i}=\left\{\begin{array}{l}
0, \text { channel } k \text { causes no interf. in cell } i \\
l, \text { channel } k \text { causes interf. in cell } i
\end{array}\right.
$$

$q_{k i}$ is used to reflect the interference between the interfering cell $i$ and cell $C$. Therefore, any available channel having minimum value of $J_{k}$ is to be allocated to a new call arising in cell $C$. To obtain an optimal reasoning, the social agents consider aggregation rules that enable the social agents to change the currently held reasoning upon acquiring new information. Information and actions that affect the social agent decision in handover process are described below.

- Handover process starts if the mobile station receives a weak SNR.

- Mobile station moves to neighbor cell that have the highest number of free channel to ensure that the call is not blocked.

- Chooses channel with the lowest interference from the set of free channels. channel $(k)=\arg \min _{k} I_{k}$

- Using iterative swapping scheme to avoid high interference.

$$
\begin{aligned}
& t=0 \\
& \text { FOR } i=1 \text { to } k \quad D O \quad r_{i}(t)=\operatorname{choose}(V) \\
& \text { REPEAT } \\
& \text { FOR } i=1 \text { to } k \quad D O \quad C_{i}=\phi \\
& \text { FOREACH } v \in V \quad D O \\
& x=\underset{i: i \in\{1, \ldots, k\}}{\arg \min } d\left(r_{i}(t), v\right) \\
& \text { ENDDO } \\
& F O R i=1 \text { to } k \quad D O \\
& r_{i}(t)=\text { minimize }\left(\operatorname{interf}\left(C_{i}\right)\right) \\
& \text { UNTIL }\left(\forall r_{i}: d\left(r_{i}(t), r_{i}(t-1)\right)<\varepsilon, \quad t>t_{\max }\right. \\
& \operatorname{RETURN}\left(\left\{r_{l}(t), \ldots, r_{k}(t)\right\}\right)
\end{aligned}
$$

\subsection{Social Agent Beliefs}

The new call in cell is blocked when there are no more free channels in the cell or the QoS requested cannot be provided as the SIR is under a given threshold $\mathrm{SIR}^{\mathrm{tgt}}$. By computing the call blocking probability in handover process, we consider four social agent decision scenarios that are described below.

1) Probability (Approve, $\mathrm{SNR}>\mathrm{SNR}^{\mathrm{tg} t}$ )

2) Probability (Reject, $S N R<S^{\text {tgt }}$ )

3) Probability(Approve, $\mathrm{SNR}<\mathrm{SNR}^{\mathrm{tgt}}$ )

4) Probability (Reject, $\mathrm{SNR}>\mathrm{SNR}^{\mathrm{tgt}}$ )

\subsection{Interference Area Identification}

In a mobile system, since the mobile stations can move between cells, the number of mobile stations within a cell at a given time can never be known exactly in advance. However, we can estimate roughly the location of the mobile stations. To avoid the call blocking by handover, it is important to identify the interference area. The interference area can be considered as the node of decision to hand over to a new cell. Denote $P_{t}$ as the transmitter power of the base station, $G$ the antenna gain, $d$ the distance between the transmitter and receiver and $N_{0}$ as the thermal noise power. Generally, then, the received signal-to-noise ratio (SNR) at the $k$-th user in cell-1 is given by

$\gamma_{1\left(d_{1}\right)}^{1}=\frac{P_{t} G\left(d_{1}\right)}{N_{0}}, \gamma_{2\left(d_{2}\right)}^{1}=\frac{P_{t} G\left(d_{2}\right)}{N_{0}}, \ldots, \gamma_{j\left(d_{k}\right)}^{1}=\frac{P_{t} G\left(d_{k}\right)}{N_{0}}(17)$ for $j=1, \ldots, k$. The signal-to-noise ratio (SNR or $\mathrm{S} / \mathrm{R}$ ) $\gamma_{k}$ defined as the ratio of a signal power to the noise power corrupting the signal. Hence, following Markovian analysis, the social agent can predict the average blocking probability of user $k$ being served by the base station based on calculation of the interference area for cell $i$, which is greater than a given target value for cell $i, \gamma_{i}^{\text {tgt }}$. This blocking probability is:

$$
P_{b}=\frac{1}{n} \sum_{k=1}^{n} \delta_{k}
$$

where $\delta_{k}=\left\{\begin{array}{ll}1 & \left(\gamma_{k}-\gamma_{i}^{\text {tgt }}\right)<0 \\ 0 & \text { Otherwise }\end{array}\right.$.

\subsection{Active Sense Environment}

In sense environment the radio cognitive approach takes in consideration the following parameters, propagation model, traffic model and amount of the information. This represents the maximum amount of information that can be conveyed through a communications channel. From an information theoretic perspective, a communications channel is responsible for passing data between two points, and will likely add some sort of noise to be original signal. In other words, the original signal reception is 
possible only when the relation of energy per bit $E_{b}$ to noise spectral density $N_{0}$ is appropriate. Low value of $\mathrm{E}_{\mathrm{b}} / \mathrm{N}_{0}$ will cause the receiver to be unable to decode the received signal, while a high value of the energy per bit in relation to noise will be perceived as interference for other users of the same radio channel. For example, for CDMA systems, the bit energy-to interference-and-noise-spectral density $\left(E_{b} / N_{0}\right)$ is SINR multiplied by the number of information bits modulated by the spreading code, whereas the carrier-to-interference-and-noise-power ratio $(C I R$ or $C / I)$ is equal to $\left(E_{b} / N_{0}\right)$ divided by the length of the spreading code. The ratio $E_{b} / N_{0}$ is given by

$$
E_{b} / N_{0}=G_{p}(C / I)
$$

where $G_{p}$ is the processing gain, and the ratio $C / I$ of the user is given by

$$
C / I=\frac{P}{E(I)}
$$

To calculate the received signal-to-noise ratio (SNR) at the $k$ th user is given by

$$
\gamma_{k}\left(r_{k}\right)=\frac{P_{t} G\left(r_{k}\right)}{N_{0}}
$$

For our purposes we focus on the Shannon Theorem, which states for additive white Gaussian noise (AWGN) channel. The channel capacity is given by

$$
C=W \log _{2}\left(1+\xi \gamma_{k}\right)
$$

where $\xi$ is the bit error rate.

\section{Simulation Results}

The radio cells treat licensed users, other unlicensed radio networks, interference, and noise all as interference affecting the signal-to-interference ratio (SIR). Higher interference yields lower SIR, which means lower capacity is achievable for a particular signal bandwidth and interference in the radio channel and reduces the quality of the transmission. There are different quantities that measure the quality as signal-to-interference ratio (SIR) and the bit-error rate (BER). SIR, referred to also as signal-to-interference-and-noise ratio (SIR) to emphasize the presence of background noise, is the ratio between the power of the desired signal and the power of the interference (plus noise). In Figure 3, two different mobile radio systems are illustrated. Mobile station $\mathrm{MS}_{\mathrm{A}}$ is located at the cell boundaries of system $A$, however very close to base station BS-B, due different reasons like receiving a weak SNR (finding the mobile station in rural area) or due interference that may occur at base station $\mathrm{BS}_{\mathrm{A}}$ from base station BS-B. To maintain a reliable connection between the user and the base station, the SIR at the receiver should be no less than some threshold that corresponds to QoS requirement such as the bit error rate. Figure 4 shows that the received signal to interference ratio varies greatly over the duration of the simulation. Figure 5 describes the average received power by the

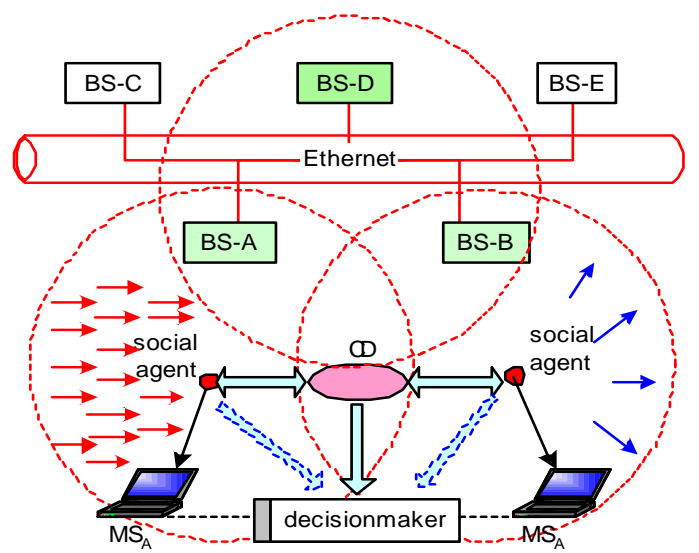

Figure 3. Traffic model.

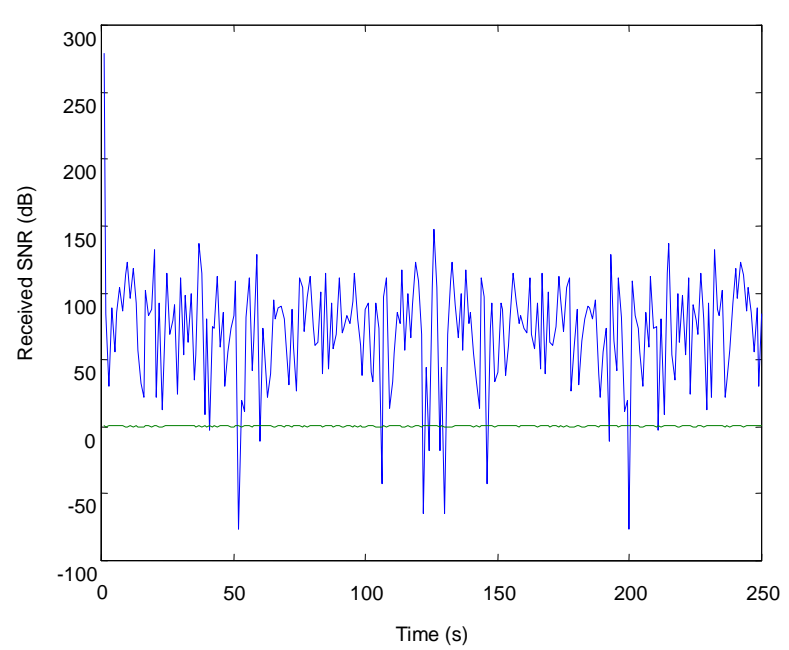

Figure 4. The received SNR.

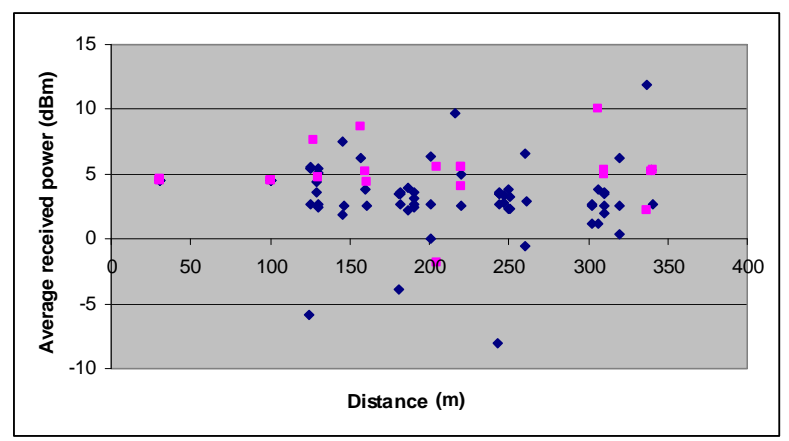

Figure 5. The received power vs. distance. 
users. Figure 6 describes the social agent strategy that may agree on a deal to exchange some of the resources they currently hold, in order to increase the social agent utility. Figure 7 describes the bandwidth efficiency. Figure 8 describes the blocking probability related to the SNR.

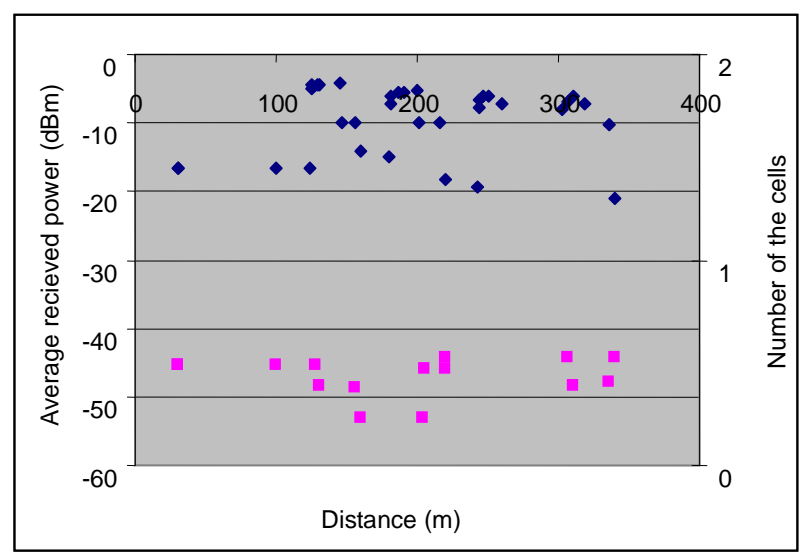

Figure 6. Resource distribution.

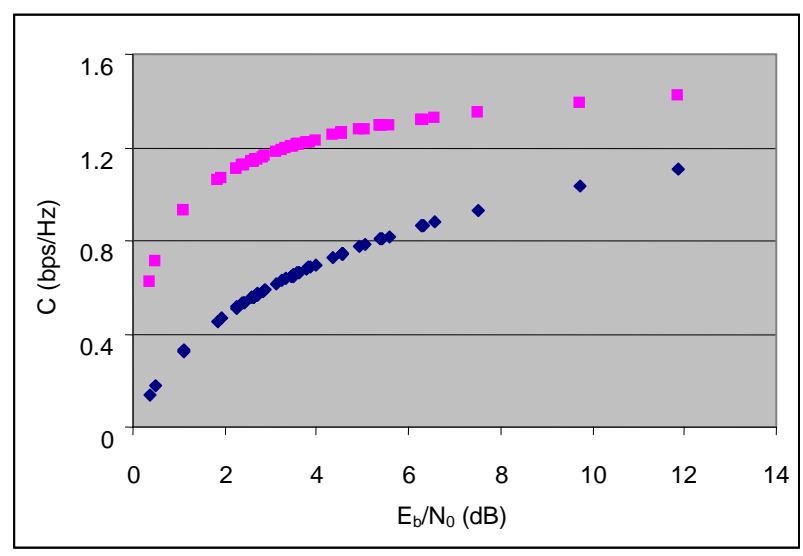

Figure 7. Bandwidth efficiency.

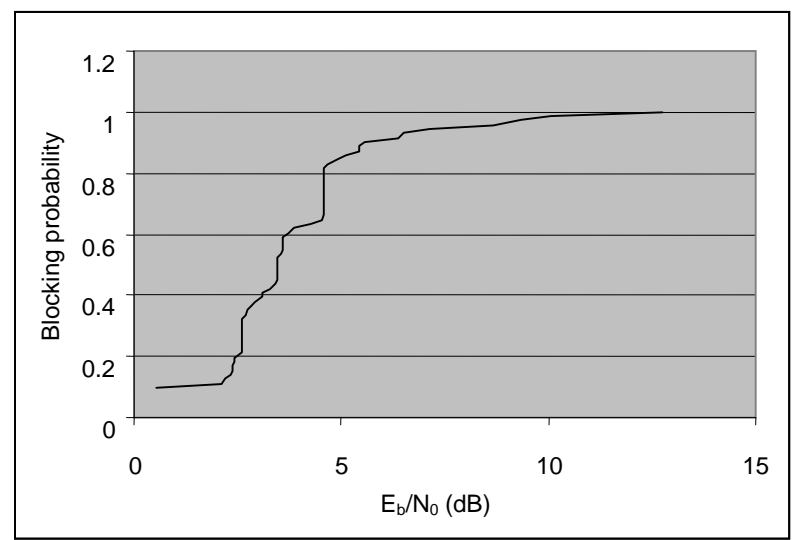

Figure 8. Blocking probability.

\section{Conclusions}

In this paper we have presented the principles of handoff procedures and described some of the procedures used in various types of systems. Furthermore we have proposed a radio cognitive for handover management to reduce the interference which is sourced by channel acquisition in cellular system. In general, there are different reasons that caused the interference in cellular system, such as power which is also an important resource. Allocation of power in the proper channels can increase capacity and avoid interference.

\section{References}

[1] N. D. Tripathi, et al., "Handoff in cellular systems," IEEE Personal Communications, December 1998.

[2] B. Friedlander and S. Scherzer, "Beamforming versus transmit diversity in the downlink of a cellular communication," IEEE Transactions on Vehicular Technology, Vol. 53, No. 53, July 2004.

[3] C. W. Leong, W. Zhuang, Y. Cheng, and L. Wang, “Optimal resource allocation and adaptive call admission control for voice/data integrated cellular networks," IEEE Transactions on Vehicular Technology, Vol. 55, No. 2, March 2006.

[4] L. Cong, et al., "Performance analysis of dynamic channel assignment algorithms in cellular mobile systems with hand-off," International Journal of Communication Systems, July 2002.

[5] S. S. Manvi and P. Venkataram, "Agent based subsystem for multimedia communications," IEE Proceedings Software, Vol. 153, No.1, February 2006.

[6] S. Anand, A. Sridharan, and K. N. Sivarajan, "Performance analysis of channlized cellular systems with dynamic channel allocation," IEEE Transactions on Vehicular Technology, Vol. 52, No. 4, July 2003.

[7] T. Ren and R. J. La, "Downlink beamformig algorithms with inter-cell interference in cellular networks," IEEE Transactions on Wireless Communications, Vol. 5, No.10, October 2006.

[8] M. Zafer and E. Modiano, "Blocking probability and channel assignment in wireless networks," IEEE Transactions on Wireless Communications, Vol. 5, No. 4, April 2006.

[9] S. Gupta and P. Srimani, "Distributed dynamic channel allocation in mobile networks: Combining search and update," The Journal Supercomputing, Vol. 17, No. 1, August 2000.

[10] S. Kandukuri and S. Boyd, "Optimal power control in interference limited fading wireless channels with outage probability specifications," IEEE Transactions on Wireless Communications, Vol. 1, No. 1, January 2002.

[11] C. J. Chen and L. W. Wang, "Impacts of radio channel 
characteristics, heterogeneous traffic intensity, and nearfar effect on rate adaptive scheduling algorithms," IEEE Transactions on Vehicular Technology, Vol. 55, No. 5, September 2006.

[12] J. Raiyn, et al., "Using adaptive agent-based on iterative distributed swapping prediction for interference reduction in cellular systems, international symposium on performance evaluation of computer and telecommunication systems," Endinburgh, U.K., 16-18 June 2008.

[13] M. Bublin, M. Kongegger, and P. Slanina, "A cost-function-based dynamic channel allocation and its limits," IEEE Transactions on Vehicular Technology, Vol. 56, No. 4, July 2007.

[14] M. S. Do, Y. Park, and J. Y. Lee, "Channel assignment with QoS guarantees for a multiclass multicode CDMA system," IEEE Transactions on Vehicular Technology, Vol. 51, pp. 935-948, September 2002.

[15] A. Seth, H. M. Gupta, and K. Momaya, "Quality of service parameters in cellular mobile communication," International Journal of Mobile Communications, Vol. 5, No. 1, pp. 65-93, 2007.

[16] S. Zhao, Z. Xiong, and X. Wang, "Optimal resource allocation for wireless video over CDMA networks," IEEE Transaction on Mobile Computing, Vol. 4, No. 1, pp. 441-424, January-February 2005.

[17] G. J. Foschini and Z. Miljanic, "A simple distribute autonomous power control algorithm and its convergence," IEEE Transactions on Vehicular Technology, Vol. 42, No. 4, pp. 641-646, November 1993.
[18] S. A. Grandhi, J. Zander, and R. Yates, "Constrained power control," Wireless Personal Communications, Vol. 1, No. 4, pp. 257-270, 1995.

[19] B. C. Jung, Y. J. Hong, D. K. Sung, and S. Y. Chung, "Fixed power allocation with nulling for TDD-based cellular uplink," IEEE Communications Letters, Vol. 12, No. 4, April 2008.

[20] X. Li and D. Wu, "Power control and channel allocation for real time applications in cellular networks," Wireless Communications and Mobile Computing, Vol. 8, No. 6, pp. 705-713, 2007.

[21] J. L. Eaves and E. K. Reely, "Principles of modern radar," Van Nostrand Reinhold Company, New York, 1987.

[22] G. J. Foschini and Z. Miljanic, "Distributed autonomous wireless channel assignment algorithm with power control," IEEE Transactions on Vehicular Technology, Vol. 44, No. 3, pp. 420-429, August 1995.

[23] D. Beckmann and U. Killat, "A new strategy for the application of genetic algorithms to channel assignment problem," IEEE Transactions on Vehicular Technology, Vol. 48, No. 4, pp. 1261-1269, 1999.

[24] M. Bergounioux and K. Kunisch, "On the structure of Lagrange multipliers for state-constrained optimal control problems," Optimization and Control of Distributed Systems, Vol. 48, No. 3-4, pp. 169-176, March 2003.

[25] S. Hykin, J. D. Thomson, and H. J. Reed, "Spectrum sensing for cognitive radio," Proceedings of the IEEE, Vol. 97, No. 5, pp. 849-877, May 2009. 